\title{
PRÁCTICUM DE LA LICENCIATURA EN PSICOLOGÍA EN LA UNIVERSIDAD ESPAÑOLA
}

\author{
PRACTICUM OF THE PSYCHOLOGY STUDIES IN \\ SPANISH UNIVERSITIES
}

\author{
José Manuel Gil Beltrán* y Esther Climent Tormos** \\ Universitat Jaume I.
}

\section{RESUMEN}

Con este trabajo se pretende mostrar como estaba el Prácticum en las Universidades Españolas hasta el curso 99/2000.

Para la realización de este estudio, se confeccionó un cuestionario, enviado al responsable de la asignatura de Prácticum de todas las universidades españolas que imparten la licenciatura en Psicología. Además de las preguntas que permitían saber la organización del Prácticum en la universidad correspondiente, la última parte del cuestionario permitía señalar propuestas de mejora para próximos cursos.

Se obtuvieron una serie de resultados entre los que destacaron los siguientes:

- La Universitat Jaume I de Castellón era la que más años llevaba impartiendo el Prácticum.

- Cada universidad oferta a sus estudiantes unas especialidades de Prácticum, y aunque se denominan de una manera concreta o se subdividen en distintos tipos, las ramas fundamentales son: Psicología Clínica, Psicología de las Organizaciones, Psicología de los Servicios Sociales, Psicología Educativa, Investigación

Palabras clave: Prácticum, aprendizaje

\section{ABSTRACT}

This work shows the situation of the practicum in Spanish universities up to the year 1999-2000.

* Gil Beltrán J.M., es Profesor Titular de Psicología evolutiva y de la Educación de la Universitat Jaume I de Castellón. Líneas de investigación: orientación y asesoramiento vocacional en E. S. Y universidad, formación de madres y padres, información vocacional, toma de decisiones y nuevas tecnologías aplicadas al asesoramiento vocacional.

** Climent Tormos E., es Técnica Superior de la Unitat de Support Educatiu de la Universitat Jaume I de Castellón. Líneas de investigación: orientación y asesoramiento vocacional en E. S. Y universidad, formación de madres y padres, información vocacional, toma de decisiones y nuevas tecnologías aplicadas al asesoramiento vocacional. 
The research was conducted using a questionnaire that was drawn up and sent to the person in charge of the practicum in all Spanish universities offering a degree in Psychology.

The questionnaire consisted of two parts: the first included all the questions concerning the organisation of the practicum in the corresponding university and the second enabled suggestions to be made for improvement in subsequent years.

The following data from the results obtained are worth mentioning:

- Universitat Jaume I in Castellón was the university with the longest history of running practicums.

- Each university offers its students different practicum specialities. Although their specific names or subdivisions may vary, the essential branches are as follows: Clinical Psychology, Organisational Psychology, Psychology of the Social Services, Educational Psychology and Research.

Key words: Practicum, learning

\section{Introducción}

La Universidad, como institución educativa, avanza para intentar dar respuesta a las demandas de los jóvenes del siglo XXI. En este avance, cabe destacar, entre otras muchas cosas, la gran importancia de las instituciones y organizaciones en las que los estudiantes realizan sus prácticas, consideradas hoy en día, como una parte importante de la formación universitaria.

Al reformarse los planes de estudio de la Licenciatura en Psicología, siguiendo la normativa de la Ley de Reforma Universitaria (LRU), se contempla por primera vez el Prácticum (estancias en prácticas) como materia troncal de la titulación, con un mínimo de 9 créditos. La calificación final de esta asignatura, no depende de ninguna otra, y tampoco está supeditada académicamente a ninguna materia específica.

El Prácticum es una propuesta educativa que requiere que el estudiante sea capaz de integrar aprendizajes de índole muy diversa. Para una buena optimización de esta asignatura se exige la acción conjunta y coordinada de las instituciones implicadas, es decir, el ámbito académico y el ámbito profesional. La universidad, debe trabajar conjuntamente con las organizaciones y instituciones que reciben estudiantes en prácticas para poder conseguir los resultados más óptimos posible. Para ello, el estudiante está supervisado por un tutor de la organización y un profesor/tutor de la titulación.

Según el BOE del 12 de enero de 1983, el Prácticum se define como: "Conjuntos integrados de prácticas a realizar en centros universitarios o vinculados a la universidad por convenios o conciertos que pongan en contacto a los estudiantes con los problemas de la práctica profesional. Podría ser también total o parcialmente de investigación. Los estudiantes realizarán un prácticum de entre los propuestos por la universidad".

No se debe entender el Prácticum simplemente como una mera realización de prácticas externas. Las prácticas deben abordarse como un trabajo universitario y requieren una importante profundización académica, el estudiante no debe ser un mero receptor de información sino que ha de ser capaz de analizar y reflexionar sobre cada momento de su experiencia práctica.

Como ya se ha comentado anteriormente, para un buen funcionamiento de la asignatura de Prácticum debe haber una buena relación entre el campo de trabajo, las materias de enseñanza que hay en la facultad y el nivel de los alumnos en su preparación educativa. El 
Prácticum debe considerarse un proyecto mutuo entre la Universidad, la empresa y los alumnos. Tal y como señalan Hopkins, Hoffman y Moss (1997), el objetivo de la coordinación entre la universidad y las organizaciones profesionales es desarrollar una comunidad de aprendizaje que pretende la implicación intelectual y el desarrollo de todos sus miembros: estudiantes, tutores externos y profesores, a través de una práctica reflexiva. Debemos considerar el Prácticum una buena forma de establecer un lazo bien atado entre la teoría y la práctica, ya que, el hecho de que los estudiantes se introduzcan en la cultura del trabajo les proporciona una idea más realista de lo que será su futuro profesional.

La propia naturaleza de la función profesional del psicólogo hace insuficiente una formación práctica de "laboratorio" desarrollada en la propia facultad. Por ello, es necesario conectar y trabajar de forma supervisada en el contexto real y futuro.

¿Qué aporta el Prácticum al estudiante? Para el estudiante, el Prácticum tiene un valor intrínseco por encima del propio valor educativo. Es muy importante para el aprendizaje del alumno el contenido de las prácticas o las dificultades encontradas en ella, pero, simplemente el hecho mismo de realizar prácticas, constituye en sí un elemento que, por tener un carácter distinto al trabajo de estudio habitual del estudiante, resulta ya una aportación relevante. Durante el tiempo que el estudiante permanece en la empresa u organización donde realiza las prácticas, tiene la posibilidad de vivir experiencias de conocimiento complementarias a las tradicionales de aprendizaje en la Universidad.

La finalidad del Prácticum está encaminada a que el alumno conozca el ámbito profesional de la Psicología, mediante un contacto directo con las organizaciones donde profesionales psicólogos ejercen su trabajo.

Como posibles objetivos del Prácticum, podemos considerar:

- Incrementar el aprendizaje y comprensión de los conceptos y habilidades enseñados en la facultad.

- Hacer que los estudiantes se motiven y encuentren significado a lo que están estudiando.

- Facilitar el desarrollo personal y social.

- Permitir que los estudiantes conozcan sus propios intereses y preferencias profesionales.

- Permitir que el estudiante tome previsiones futuras de la profesión.

Concretamente, con el Prácticum se trata de ofrecer a los estudiantes la posibilidad de observar y analizar algunos de los contextos en los cuales en un futuro podrá llevar a cabo su trabajo, de manera que puedan tener un conocimiento real del trabajo profesional y de las diferentes metodologías usadas, así como de todos los aspectos que integran la actividad profesional (servicios, programas, organización, funciones, etc.) y las características de las personas o de los diferentes grupos objeto de intervención.

Para el desarrollo del Prácticum se necesita de programas que faciliten el aprendizaje de la práctica y proponga situaciones de aprendizaje de la experiencia dentro de un contexto profesional específico.

El hecho de que los estudiantes cursen la asignatura de Prácticum posibilita que ganen confianza en sí mismos. A pesar de los años que llevan estudiando y de toda la formación teórica que reciben, la mayoría de ellos, demuestra sensación de inseguridad profesional al acabar los estudios. Mediante la realización de las prácticas, los estudiantes constatan que 
no saben tan poco como creen y son capaces de poner en práctica los conocimientos teóricos que ayudan en un futuro profesional a ganar confianza.

Para el desarrollo del Prácticum son fundamentales dos figuras:

- Profesor-tutor de la titulación.

- Tutor en la empresa u organización.

a) Profesor-tutor de la titulación

El tutor de la titulación es el profesor universitario encargado de la relación directa con los alumnos y desempeña un rol de orientación práctica y ayuda a los estudiantes.

El profesor-tutor de las prácticas debe también asumir la función de asesor y entrenador de habilidades sociales, tales como escuchar, comunicarse, saber acoplarse a diversas estructuras, saber aportar opiniones, etc. El carácter de la acción tutorial debe ser de cooperación, no sólo con el estudiante sino también con los profesionales del campo práctico. La asignatura de Prácticum es un proceso en el que intervienen tres partes: estudiante, Universidad y entidad laboral, y una de las funciones de este tutor es preocuparse de que en este proceso exista intercambio y diálogo entre las tres partes. Este intercambio es necesario para asegurar la comunicación entre el contexto académico y el contexto laboral. Es considerado también requisito indispensable para el aprendizaje integrado del estudiante y condición necesaria para que el Prácticum sea una ocasión en la que la Universidad y el mundo laboral reflexionen sobre su propia identidad y sus relaciones mutuas.

b) Tutor en la empresa u organización

El tutor en la empresa u organización es un profesional que trabaja en la empresa y está encargado de la formación de los estudiantes.

Las prácticas no deben ser supervisadas únicamente por el profesor universitario, es necesaria la figura del experto en su propio campo profesional. Este tutor es capaz de crear unas oportunidades internas al propio campo de trabajo y la comprensión del mundo profesional, y proporcionar a los estudiantes un conocimiento práctico y una mejor comprensión de lo que será su futuro trabajo. Según Bell (1997), los tutores de la empresa que son eficaces "son como amigos" teniendo en cuenta que su objetivo es crear un contexto seguro para el crecimiento.

Las funciones principales que tiene el tutor en la empresa puede considerarse que son:

- recibir a los alumnos e informarles del funcionamiento general de la empresa

- explicar a los alumnos los actividades que deben desarrollar

- supervisar la asistencia, actitud, participación y rendimiento de los alumnos

- atender a las consultas teóricas o prácticas de los alumnos que tengan relación con el trabajo que él desempeña como profesional de la empresa

- crear oportunidades para la observación que sirvan al mismo tiempo para enseñar

- crear oportunidades para que puedan trabajar con otro tipo de profesionales del mismo departamento o empresa

- proporcionar apoyo y seguridad. 


\section{Método}

Para la realización del estudio del Prácticum en Psicología en la Universidad Española se confeccionó un cuestionario (ver anexo 1) con el que se pretendía averiguar en qué consistía y cómo estaba organizado el Prácticum en las distintas universidades españolas. El cuestionario está organizado de la siguiente manera:

$\mathrm{Al}$ principio se les piden los datos identificativos y un teléfono y dirección de correo electrónico para un más fácil contacto. Las preguntas del cuestionario se pueden dividir en tres partes: en primer lugar, se les piden datos de descripción de la asignatura de Prácticum en su Universidad (ejemplo: años que llevan impartiéndola, tipos de Prácticum, ...) — preguntas 1, 2, 3 y 4-; en segundo lugar, se abordan cuestiones para conocer cómo está organizada la asignatura, es decir, quién realiza la adjudicación de plazas, qué tipos de convenios tienen, si realizan trabajos o memorias de evaluación, etc. - preguntas 5, 6, 7, 8, 9, 10, $11,12,13,14$ y 15-; finalmente, las últimas cuatro preguntas $(16,17,18$ y 19) se centran en la valoración de la asignatura por parte de estudiantes y tutores de empresas y en las dificultades que se encuentran al implementar la asignatura, así como también en qué propuestas de mejora harían para mejorarla.

\section{Procedimiento}

Se contactó bien por teléfono, bien por correo electrónico con cada universidad y se envió el cuestionario al responsable del Prácticum de Psicología de la universidad correspondiente vía e-mail y vía correo tradicional (curso 2000/2001).

Un total de ocho universidades fueron las que contestaron el cuestionario y con las que se realizó el estudio: Universidad de Sevilla; Universidad Pontificia Comillas de Madrid; Universidad de Murcia; Universidad de Deusto; Universidad Autónoma de Madrid; Universidad de Oviedo; Universidad de Santiago de Compostela; Universitat Jaume I de Castellón.

\section{Resultados}

A continuación se van a presentar los resultados obtenidos a partir del análisis del cuestionario y se comparan las semejanzas y diferencias encontradas en las distintas Universidades Españolas respecto a la asignatura de Prácticum hasta el curso 1999/2000.

Con los datos extraídos de las preguntas de la primera parte del cuestionario, podemos observar que:

La Universitat Jaume I, hasta ese momento, lleva ocho años impartiendo la asignatura de Prácticum, lo cual nos indica que de las Universidades de las cuales tenemos información es la que más años lleva haciéndolo, la Autónoma de Madrid y de la de Oviedo desde hace cinco años; la Universidad Pontificia Comillas de Madrid, cuatro años, la Universidad de Deusto y la de Santiago de Compostela, tres años, la de Sevilla, dos años y finalmente, la que menos tiempo lleva impartiéndola es la Universidad de Murcia, tan sólo hace un año. 


\begin{tabular}{|l|c|}
\hline \multicolumn{1}{|c|}{ UNIVERSIDAD } & $\begin{array}{c}\text { AÑOS IMPARTIENDO } \\
\text { PRÁCTICUM }\end{array}$ \\
\hline Universitat Jaume I & 8 \\
\hline Universidad Autónoma de Madrid & 5 \\
\hline Universidad de Oviedo & 5 \\
\hline Universidad Pontificia Comillas de Madrid & 4 \\
\hline Universidad de Deusto & 3 \\
\hline Universidad Santiago de Compostela & 3 \\
\hline Universidad de Sevilla & 1 \\
\hline Universidad de Murcia & 2 \\
\hline
\end{tabular}

Cada Universidad oferta a sus estudiantes unos determinados perfiles o especialidades de Prácticum, en función de las posibilidades que tienen de ofertar plazas. Aunque cada Universidad los denomina de una manera o los subdivide en distintos tipos, las ramas fundamentales que ofertan son:

- Psicología Clínica.

- Psicología de las Organizaciones.

- Psicología de los Servicios Sociales.

- Psicología Educativa.

- Investigación.

La Universitat Jaume I oferta un total de 17 especialidades de Prácticum que muchas de ellas se pueden encuadrar en las líneas anteriores, pero como Prácticums a destacar, considerándolos notas diferenciales respecto a otras Universidades estarían:

- Psicobiología Clínica: Planificación y Terapias Sexuales.

- Psicología Forense y Penitenciaria.

- Informes y Peritajes en Psicología.

Para el resto de Universidades, cabe destacar como una característica diferencial que la Universidad de Deusto oferta el Prácticum de Investigación Individual para aquellos alumnos que no pueden comprometerse con horarios o exigencias o a aquellos que no han aprobado el primer ciclo de estudios.

La Universidad Autónoma de Madrid, añade a los anteriores un Prácticum en Metodología y un Prácticum en Psicología Ambiental, teniendo en cuenta que éste último es la única Universidad que lo oferta. Señalar también que el Prácticum en Psicología del Deporte es sólo la Universidad de Oviedo la que lo ofrece a sus estudiantes.

Como nota diferencial de la Universidad de Santiago de Compostela, tenemos el Prácticum en Psicología Básica y el Prácticum en Psicobiología.

En todas las Universidades la asignatura de Prácticum es una asignatura troncal pero varía el número de créditos que los estudiantes tienen que cursar. La Universitat Jaume I 
hace necesario que sus estudiantes cursen un total de 32 créditos, con lo cual se sitúa a la cabeza del resto de Universidades, seguida de la Universidad Autónoma de Madrid con 16, la de Oviedo con 15 y la de Sevilla con 12 créditos. En el resto de Universidades encuestadas (Pontificia Comillas de Madrid, Murcia, Deusto y Santiago de Compostela), la asignatura de Prácticum sólo tiene una duración de 90 horas, es decir, 9 créditos. Cabe destacar que en la Universidad Pontificia Comillas de Madrid, los nueve créditos se realizan la mitad en cuarto curso y la otra mitad en quinto.

\begin{tabular}{|l|c|}
\hline \multicolumn{1}{|c|}{ UNIVERSIDAD } & CRÉDITOS \\
\hline Universitat Jaume I & 32 \\
\hline Universidad Autónoma de Madrid & 16 \\
\hline Universidad de Oviedo & 15 \\
\hline Universidad Pontificia de Comillas & 9 \\
\hline Universidad de Deusto & 9 \\
\hline Universidad de Santiago de Compostela & 9 \\
\hline Universidad de Sevilla & 12 \\
\hline Universidad de Murcia & 9 \\
\hline
\end{tabular}

Al analizar la segunda parte del cuestionario, se obtienen conclusiones como las siguientes:

En todas las Universidades españolas, todos los estudiantes cumplen con el total de créditos realizando las horas en las empresas o instituciones; el resto de tareas que tienen que realizar no se computan como horas de Prácticum. Las excepciones son la Universidad de Deusto que reserva un crédito para una sesión de orientación inicial en la Universidad y otro para las tutorías universitarias individuales o grupales y la Jaume I que en algunos tipos de Prácticum reserva algún crédito para seminarios.

La Universitat Jaume I, la Universidad de Murcia y la Universidad de Oviedo, cuentan con una persona responsable de las estancias en prácticas de todas las carreras de la Universidad. El resto de universidades encuestadas, hasta ese momento, no tienen un responsable de prácticas en la misma línia que las anteriores.

Todas las universidades que respondieron el cuestionario, tienen una persona responsable de la asignatura de Prácticum y en la mayoría cada especialidad del Prácticum tiene un coordinador.

Todas las Universidades encuestadas, ofrecen a sus estudiantes una guía sobre la asignatura de Prácticum en la cual les ofrecen información sobre aspectos como:

- Definición del Prácticum.

- Organización del Prácticum.

- Lugares donde pueden realizar el Prácticum.

- Estudiantes que pueden matricularse en la asignatura de Prácticum. 
- Tutores del Prácticum.

- Tareas del estudiante una vez matriculado en el Prácticum.

- Calificación de la asignatura de Prácticum.

Todas las Universidades encuestadas, realizan una memoria de evaluación del Prácticum, aunque hay que decir que no en todas son los profesores/tutores los que realizan esta memoria, sino que en alguna son comisiones o delegaciones del Prácticum como en la de Sevilla o en la Autónoma de Madrid. Algunos aspectos que incluyen estas memorias de evaluación son: problemas surgidos, valoración de los centros, valoración de la actividad del alumno, etc.

Los tutores de las empresas realizan siempre alguna evaluación del estudiante que han estado tutorizando, exceptuando los pertenecientes a la Universidad Pontificia Comillas de Madrid que no realizan ningún trabajo o memoria de evaluación. Los alumnos de esta Universidad tampoco realizan ninguna memoria; el resto de Universidades si que exigen a sus alumnos que realicen una memoria que, aunque depende de muchos factores, tiende a incluir las labores que allí han desempeñado y una valoración de la experiencia.

Para que los estudiantes puedan realizar las prácticas en las empresas o instituciones correspondientes, todas las Universidades tienen algún tipo de convenio con ellas (convenios de cooperación educativa, de colaboración, etc.).

Los alumnos eligen el sitio o la especialidad del Prácticum que quieren cursar, pero son los profesores los que acaban adjudicándoles el puesto más idóneo, según su preferencia, especialidad o los requisitos que demandaba la empresa. En algunas Universidades como la Jaume I o la Autónoma de Madrid, los estudiantes hacen una preinscripción antes de matricularse en la asignatura.

La tercera parte del cuestionario, en la que se preguntaba sobre cómo valoraban la asignatura, las dificultades que estaban encontrando y las propuestas de mejora, nos hace llegar a las siguientes conclusiones:

La valoración que hacen, tanto los estudiantes como los tutores de las empresas, de la experiencia del Prácticum es positiva según la opinión de las Universidades encuestadas. Cabe destacar que la Universidad de Murcia, a pesar de la valoración positiva por ambas partes, añade que siempre hay estudiantes que critican que se les manden tareas no propias del psicólogo y que las empresas demandan más apoyo técnico por parte de la facultad.

Exceptuando la Universidad de Sevilla que no señala ninguna dificultad a la hora de implementar la asignatura de Prácticum, el resto de Universidades si consideran que tienen alguna que otra dificultad. Destacan el excesivo número de alumnos en universidades como la de Santiago de Compostela o la Autónoma de Madrid, pero en general, las dificultades que más señalan son: el encontrar centros y el que la Universidad tenga una mejor reconocimiento de los tutores de las empresas y no se tenga que depender de la buena voluntad de los psicólogos.

Tres Universidades proponen para una mejora de la asignatura de Prácticum que se aumente el número de créditos (Universidad de Sevilla, Universidad de Deusto y Universidad de Santiago de Compostela). Tanto la Universidad Pontificia Comillas de Madrid, como la de Murcia, la Autónoma de Madrid y la de Oviedo consideran necesario que haya mayores recursos económicos y humanos para la implementación de esta asignatura. También desta- 
can la Universidad de Murcia y la de Oviedo el hecho que las estancias en prácticas distorsionan el ritmo de las clases en la facultad. En la Universidad de Oviedo ya han solucionado este problema, reduciendo la carga lectiva del último año de la licenciatura.

La Universidad de Deusto considera importante que formaran parte de la asignatura procesos de tutoría que permitieran a los estudiantes una mejor reflexión sobre la práctica.

La Universidad Autónoma de Madrid piensa que deberían realizarse más y mejores convenios, crear oficinas de Prácticum con personal estable responsable de la gestión y promover servicios o centros de Psicología Aplicada anexos a las facultades para que los alumnos tengan otra opción a la hora de realizar el Prácticum, además esto facilitaría el control y seguimiento de las prácticas.

Finalmente, la Universitat Jaume I, cree que habría que objetivarse los criterios de evaluación de esta asignatura y ampliar la carrera en un año, para que el solapamiento con clases teóricas sea menor.

\section{Discusión}

Con todo lo expuesto en el trabajo, cabe destacar la importancia y el valor fundamental de las prácticas para cualquier tipo de aprendizaje y en concreto, para el aprendizaje y la consolidación de conocimientos de cualquier rama de la Psicología.

En 1968 se estableció la Licenciatura en Psicología, pero se hizo siguiendo la misma estructura que había tenido hasta entonces dentro de la facultad de Filosofía, es decir, dos años de estudios comunes y tres años de especialización en Psicología. Ya en la redacción de este primer plan de estudios encontraron fundamental que las prácticas tenían que tener un lugar y un papel importantes, no sólo en aquellas asignaturas como la estadística o el psicodiagnóstico, sino también para otras muchas en las que se necesitaban sujetos y contextos reales.

La asignatura de Prácticum aparece como novedad en 1983 cuando se redacta la Ley de Reforma Universitaria. A partir de ese momento es considerada una materia dedicada completamente a que los estudiantes entren en contacto en un marco de aprendizaje real dentro de un contexto profesional donde tendrán que desempeñar su trabajo en un futuro.

La asignatura de Prácticum no debemos entenderla simplemente como una mera realización de prácticas externas. Esta estancia en prácticas ha de entenderse como cualquier otra asignatura universitaria y requiere, evidentemente, una importante profundización académica; el estudiante ha de ser capaz de analizar e investigar cada momento de su experiencia práctica.

Entendemos por Prácticum siguiendo el BOE del 12 de enero de 1983: "Conjuntos integrados de prácticas a realizar en Centros Universitarios o vinculados a la Universidad por convenios o conciertos que pongan en contacto a los estudiantes con los problemas de la práctica profesional. Podría ser también total o parcialmente de investigación. Los estudiantes realizarán un Prácticum de entre los propuestos por cada Universidad".

Con esta asignatura pretendemos objetivos como:

- Permitir que los estudiantes comprendan mejor los conceptos teóricos y las habilidades que han aprendido o están aprendiendo en la universidad. 
- Aumentar la motivación de los estudiantes.

- Ayudar a los estudiantes a desarrollarse personal y socialmente.

- Darles a conocer y que lleguen a comprender el mundo laboral.

- ...

Para el desarrollo de la asignatura de Prácticum, no basta con que el estudiante acuda a la empresa o institución, sino que un trabajo previo y continuado durante la estancia, será necesario para un buen aprovechamiento de ésta. Son fundamentales la figura del tutor en la universidad así como también la del tutor de la empresa.

El tutor en la universidad es un profesor universitario encargado de velar para que haya un buen intercambio y diálogo entre el contexto académico y el contexto laboral y ayudar a que el estudiante aproveche su estancia y cumpla al máximo los objetivos propuestos.

El tutor de la empresa es un experto en su campo profesional que es el encargado de supervisar al estudiante en todas las actividades y tareas que tiene que realizar dentro de la empresa, es decir, se ha de corresponsabilizar de la formación de los estudiantes dentro de la empresa.

Es importante destacar que en el análisis de las universidades españolas destaca como una de las principales dificultades el hecho de que no haya un mejor reconocimiento de los tutores de las empresas; en general, todas las universidades proponen que debería haber mayores recursos económicos y humanos para la implementación de esta asignatura.

La Universitat Jaume I fue pionera en la implantación de la asignatura de Prácticum a la vista de los datos que tenemos de otras universidades, la está impartiendo desde el curso 92/93, y hay universidades como la de Murcia que en el curso 98/99, acababan prácticamente de ponerla en funcionamiento.

Con todos los datos analizados y explicados en el punto anterior, podemos observar como la Universidad Española apuesta por la formación práctica de los futuros psicólogos/as y se puede llegar a la conclusión de que las Prácticas en contextos reales son un instrumento eficaz que no hay que descartar ni cambiar por nada, y además, si se complementan con una actitud abierta, activa y reflexiva por parte del estudiante, formarán un conjunto que será la clave perfecta para un aprendizaje eficaz.

\section{Referencias bibliográficas}

Asrs, F. (1996): “¿Prepara la Universidad para el ejercicio profesional?”. Papeles del Colegio, 66, 8790.

Balsells, A.; Jové, G. y Noria, M. (1998): "El Prácticum: instrumento de reflexión en y de la práctica". En González, M. N., Lobato, C. y Ruiz, M. P. Desarrollo Profesional y Prácticum en la Universidad. Bilbao, pp. 225-232.

Berenguer, G. y Quintanilla, I. (1994): "La imagen de la psicología y los psicólogos en el estado español”. Rev. Papeles del Psicólogo, 58, pp. 41-68.

Bisquerra, R. y Álvarez, M. (1997): "Los modelos de orientación e intervención psicopedagógica en la formación de los orientadores: algunas reflexiones sobre el estado de la cuestión". La orientación educativa y la intervención psicopedagógicas integradas en el vitae. AEOP, Valencia. 
Blanco, A. (1998): "Requisitos y necesidades de formación para la psicología del siglo XXI". Rev. de Psicología General y Aplicada, 51 (1), pp. 149-172.

Blanco, A. y Botella, J. (1995): "La Enseñanza de la Psicología en España a la luz de los nuevos planes de estudio". Rev. Papeles del Colegio, 62, pp. 29-47.

Blas, F. (1996): “¿Prepara la Universidad para el ejercicio profesional?”. Rev. Papeles del Colegio, 66, pp. 87-90.

Cáceres, M. (1998): "Prácticum, coordinación y seguimiento. Ventajas e inconvenientes". En González, M. N.; Lobato, C. y Ruiz, M. P. Desarrollo Profesional y Prácticum en la Universidad. Bilbao, pp. 349-354.

Cifuentes, Ma A. (1997): "Una reflexión sobre las prácticas". Rev. Interuniversitaria de Formación del Profesorado, 30, pp. 161-175.

Climent, E. y Gil, J. M.(2000): El psicólogo en el siglo XXI. Papel del Prácticum en su formación. Tesis de Licenciatura. Universitat Jaume I. Castellón.

Coll, C. (1989): "Psicología académica y psicología profesional en el campo de la educación". Rev. Anuario de Psicología, 41, pp. 49-73.

Crespo, J. (1997): "Nuevos retos en la formación profesional del orientador". La orientación educativa y la intervención psicopedagógicas integradas en el currículum. AEOP, Valencia.

Cunningham, J. and Oakland, T. (1998): "International School Psychology Association Guidelines for the Preparation of School Psychologists". Rev. School Psychology International, Vol. 19, 1.

Galarreta, J.; Martínez, B.; Orcasitas, J. R. y Pérez-Sostoa, V. (1998): "El grupo de seguimiento como contexto de aprendizaje en la supervisión del Prácticum". En González, M. N., Lobato, C. y Ruiz, M.P. Desarrollo Profesional y Prácticum en la Universidad. Bilbao, pp. 135-143.

Hopkins, W. S.; Hoffman, S. Q. and Moss, V. D. (1997): "Professional Development Schools and Preservice Teacher Stress". Action in Teacher Education, Vol. 18, 4, pp.36-46.

Rodríguez, M. L. (1999): Enseñar a explorar el mundo del trabajo. Diagnóstico de las destrezas exploratorias y propuestas de intervención. Ediciones Aljibi. Málaga.

Senge, P. (1995): La Quinta Disciplina en la Práctica. Barcelona, Granica.

Siguan, M. (1978): "La enseñanza universitaria de la psicología en España. Notas para su historia". Rev. Anuario de Psicología, 19, pp. 127-137.

Siguan, M. (1989): "Enseñanza universitaria y ejercicio profesional de la psicología". Rev. Anuario de Psicología, 41, pp. 5-21.

Torrego, J. C. y García-Fraile, F. (1997): "Formación de profesionales de la orientación en la Comunidad de Madrid". En La orientación educativa y la intervención psicopedagógicas integradas en el currículum. AEOP, Valencia.

Veenman, S. (1984): "Perceived problems of beginning teachers". Review of Educational Research, vol 54, 2, pp. 143-178.

Vélez, E. y Moreno, E. (1997): "Una propuesta de desarrollo profesional a través de la formación en adaptaciones curriculares de equipos docentes". Rev. Interuniversitaria de Formación del Profesorado, 30, pp. 151-159.

Vezzetti, H. (1998): "Las Psicologías del fin de siglo a la luz de su historia". Rev. de Psicología General y Aplicada, 51 (1), pp. 105-114.

Yela, M. (1994): "La enseñanza de la Psicología en España". Rev. Papeles del Colegio, 60, pp. 39-48.

Zabalza, M. A. (1998): "Evaluación del Prácticum (Diseño de la evaluación, puesta en práctica, valoración, problemas y limitaciones)". En González, M. N., Lobato, C. y Ruiz, M. P. Desarrollo Profesional y Prácticum en la Universidad. Bilbao, pp. 55-62. 


\section{ANEXO 1 \\ Cuestionario sobre la asignatura de Prácticum en la licenciatura en Psicología}

UNIVERSIDAD:

NOMBRE DEL PROFESSOR QUE CONTESTA EL CUESTIONARIO:

Teléfono:

E-mail:

1. ¿Cuántos años llevan impartiendo la asignatura de Prácticum en esa Universidad?

2. ¿Qué tipos de Prácticum imparten?

$\square$ Psicología Clínica

$\square$ Psicología de las Organizaciones

$\square$ Psicología de los Servicios Sociales

$\square$ Psicología del Deporte

$\square$ Psicología Educativa

$\square$ Otros (Especifique lo más claramente posible)

3. ¿De qué carácter es la asignatura de Prácticum (troncal, obligatoria, optativa?

4. ¿De cuántos créditos consta la asignatura de Prácticum?

5. ¿Realizan los alumnos todo el número de créditos en la institución correspondiente? $\square$ SI $\square$ NO

6. ¿Se reserva un número determinado de créditos para otras tareas, como por ejemplo, reuniones con tutor en la universidad, realización de memoria, etc.) $\quad \square$ SI $\square$ NO En caso de que se reserven créditos para este tipo de tareas, especificar concretamente para qué tipo:

7. ¿Hay alguna persona responsable de las estancias en prácticas de todas las carreras de la universidad? SI/NO

Nombre:

Telf. Contacto: E-mail: 
8. ¿Hay un profesor responsable de la asignatura de Prácticum en Psicología que se encarga de la coordinación? $\square$ SI $\square$ NO

Nombre:

Telf. Contacto: E-mail:

9. ¿Tienen algún tipo de guía para los estudiantes sobre la asignatura de Prácticum que les sirva a la hora de matricularse? $\square$ SI $\square$ NO

En caso afirmativo, qué tipo de información ofrece la guía:

Definición del Prácticum

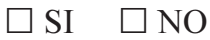

Organización del Prácticum

$\square$ SI $\square$ NO

Lugares donde puede realizarse el Prácticum

$\square$ SI $\square$ NO

Estudiantes que pueden matricularse en el Prácticum

$\square \mathrm{SI} \quad \square \mathrm{NO}$

Tutores del Prácticum

$\square$ SI $\square$ NO

Tareas del estudiante una vez matriculado

$\square$ SI $\square$ NO

Calificación de la asignatura de Prácticum

$\square$ SI $\square$ NO

Otras:

10. ¿Se realiza por parte de los profesores/tutores de la universidad una memoria de evaluación del Prácticum? $\square$ SI $\square$ NO

¿Qué tipo de información aporta?

11. ¿Realizan los tutores de las empresas o instituciones algún tipo de memoria de evaluación? $\square$ SI $\square$ NO

¿Qué tipo de información contiene?

12. Los alumnos, ¿realizan algún trabajo o memoria de evaluación de sus estancias en prácticas? $\square$ SI $\square$ NO

En caso afirmativo, ¿qué tipo de información incluye?

13. ¿Qué tipo de convenios realiza la universidad con las empresas o instituciones colaboradoras? 
14. Los alumnos, ¿pueden elegir el sitio donde quieren realizar las prácticas en función de lo que se les oferta? $\quad \square$ SI $\quad \square$ NO

15. ¿Son los profesores los que siguen algún otro criterio a la hora de asignarles el puesto? $\square$ SI $\square$ NO

En caso afirmativo, ¿qué tipo de criterio?

16. ¿Qué valoración suelen hacer los estudiantes de su estancia en prácticas?

17. ¿Qué valoración suelen hacer los tutores de las empresas o instituciones de la estancia en prácticas de alumnos universitarios?

18. Indique qué dificultades encuentran ustedes a la hora de poder implementar la asignatura de Prácticum (excesivo $\mathrm{n}^{\mathrm{o}}$ de estudiantes, falta de instituciones o empresas, ...)

19. ¿Qué propuestas de mejora realizaría usted respecto a la asignatura de Prácticum?

Fecha de recepción: 25-05-04

Fecha de revisión: 27-04-05

Fecha de aceptación: 19-10-05 